\title{
Design of a Monitoring and Test system for PV based Renewable Energy Systems
}

\author{
S. Berberkic, P.J. Mather, V. Holmes and M. Sibley \\ School of Computing and Engineering \\ SERG, Huddersfield University \\ Queensgate, HD1 3HD Huddersfield (UK) \\ Phone:+ 44(0)1484473524, e-mail: S.Berberkic@ hud.ac.uk
}

\begin{abstract}
To optimize the energy production and usage, of a Renewable Energy System (RES), it is essential that an effective monitoring and control strategy is employed. In order to develop new control algorithms the designer needs to evaluate the RES parameters and at the same time simulate real test condition load profiles.

The developed system is composed of a monitoring tool that measures and displays, in real-time, the parameters of PhotoVoltaic (PV) solar panels, and of an electronic load that can simulate load profiles such as the averaged domestic or stepped power consumption.

The monitoring system is implemented using a $\mu$ controller and the electronic load is composed of a MOSFET, driven using a Pulse Width Modulated (PWM) output of a PIC $\mu$ controller. Furthermore, the power consumption profiles are stored in the internal memory of the PIC $\mu$ controller.

The obtained results demonstrate the ability of the system to monitor the current, voltage and temperature of PV panels as well as the ability to simulate different electronic load profiles.
\end{abstract}

\section{Key words}

Renewable energy, monitoring, electronic load.

\section{Introduction}

At present, world energy production is mainly based on the use of limited resources, namely fossil fuels. With the ever increasing demand for energy, some predictions show that world oil reserves will be exhausted by 2030 or at best by 2100 if the consumption is radically reduced from present day levels. Given that the oil is the main resource for energy production, it is obvious that new energy sources need to be developed. However, new sources of energy need to be cost effective and sustainable [1]. The research into sustainable energy sources includes nuclear power production as well as other unlimited energy sources such as wind, tidal, biomass and solar energies [2]. While the sustainability factor is important when developing Renewable Energy Sources (RES), the economic factor remains the key to the large scale adoption of a technology. It is important to note that the economic viability of the system is directly linked to the overall system efficiency; a good example being PhotoVoltaic (PV) solar installation payback time [4]. The energy efficiency and optimized usage are important, when developing and designing RES systems, hence many monitoring systems have been developed [7-8]. However the main focus, of this work, is the monitoring and control of energy production for a PV installation. The power from a PV system can reduce significantly if the connected load is modified [3]. Hence, an effective load control strategy plays an important role in the system efficiency [5].

For a fixed PV system there are two possible ways of increasing the efficiency:

$>$ Improving the control strategy.

$>$ Modifying the load profile (demand side management)

In both cases the designer requires a reliable monitoring tool in order to assess the overall system efficiency as well as an electronic load capable of simulating the desired load profile. Furthermore, the load profile for the RES needs to be set to simulate real applications, thus a test bed is an effective technique for testing control strategies in hybrid RESs [6]. Hence the aim of this research is to design and develop such a monitoring and test system.

\section{System Description}

The developed system is composed of three modules; firstly the acquisition module, which is physically connected to the RES under test, through a series of sensors. Once the different sensor signals are digitalized and processed the data is then sent to the PC interface module, in order to be displayed, on a PC, using a LabVIEW graphical user interface. The PC interface module is also fitted with a LCD to allow remote monitoring and also data access when the PC is off. The data transition between the two modules is undertaken using IEEE 802.15.4-2003 standard ZigBee devices. 
Finally, the electronic load module is controlled and powered by the acquisition module.

The system block diagram is shown in fig. 1 .

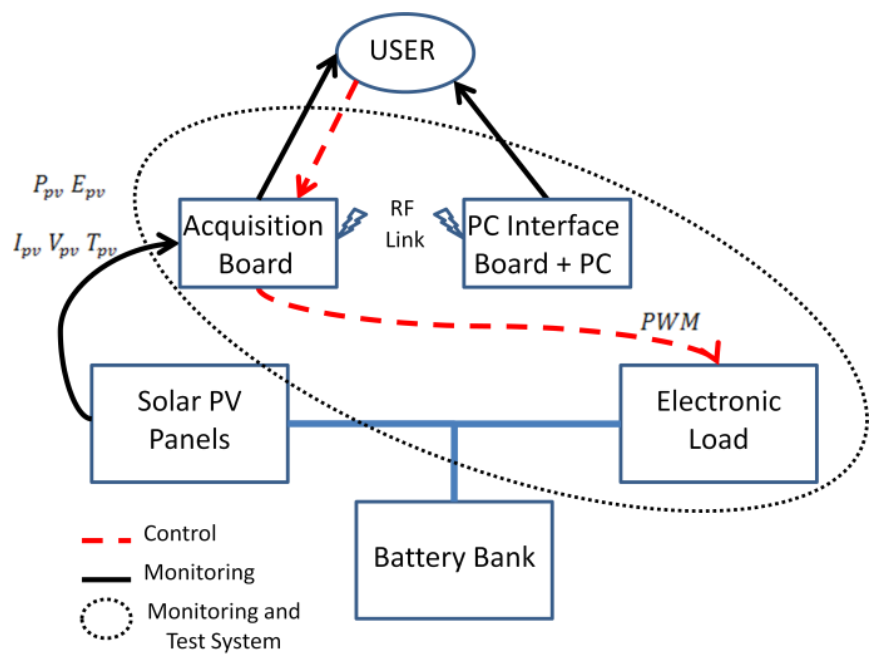

Fig. 1: System Block Diagram

\section{A. Acquisition}

The acquisition module is composed of sensors for the measurement of current, voltage and temperature. The sensor signals are conditioned using analogue circuitry and sampled by a PIC16F690 $\mu$ controller. The signal processing circuitry for current measurement is shown in fig. 2 .

The system specifications to be monitored are the DC Voltage and Current measurements as well as cell temperature.

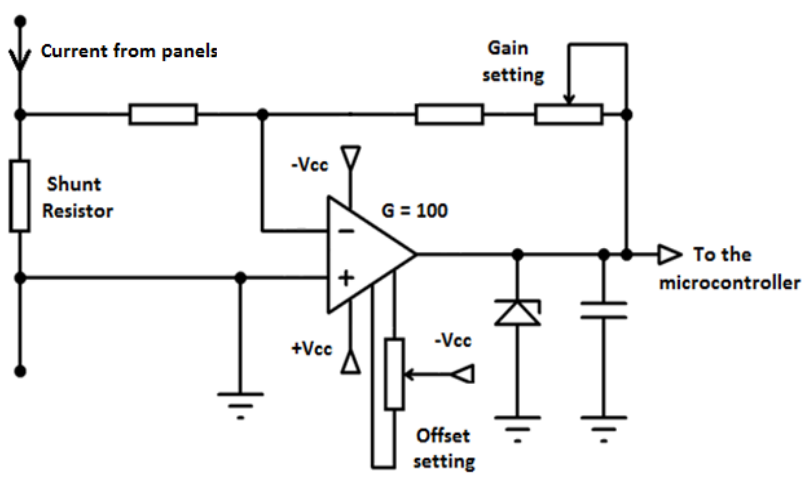

Fig. 2: Current Measurement Conditioning Circuitry.

Assuming that the PV panels are used to charge $12 \mathrm{~V}$ or $24 \mathrm{~V}$ batteries, a $30 \mathrm{~V}$ measurement range was selected. The current measurement range was set to $0-50 \mathrm{~A}$, which is appropriate for small to medium sized arrays. However, a smaller 0- 5A range is also available by changing the shunt resistor. The cell temperature measuring range was set to $0-150^{\circ} \mathrm{C}$.

The instantaneous power $\left(P_{p v}\right)$ of the system is displayed from 0 to $1.5 \mathrm{~kW}$ and is calculated using the instantaneous voltage and current values. The energy $\left(E_{p v}\right)$ is calculated using $P_{p v}$ and the running time and has a range of 0 to $999 \mathrm{kWhr}$. The data is transmitted from the acquisition module to the PC interface via a wireless link, since the PV panels are generally situated on a roof and the PC in the house. The wireless link was implemented using Zigbee Pro modules to allow reliable transmission of data.

Finally, a Pulse Width Modulated (PWM) signal is generated by the acquisition module to control the electronic load. The different load profiles are stored in the internal memory of the PIC with three main choices available; constant power, stepped and a typical household averaged load profile. More details about the different load modes are given in the 2. $C$ section.

\section{B. PC Interface}

The PC interface receives data from the acquisition module and forwards it to the PC using the RS-232 serial protocol. The PC displays the data using LabView and a PC interface LCD display enables data reading when the $\mathrm{PC}$ is turned off.

The user interface needs to be easy to understand and interpret, thus the following specifications were set:

$>$ Display digital values and visual representation for Current, Voltage, Temperature and Power.

$>$ Display Temperature using a visual thermometer representation.

$>$ Alert the user with visual and audio signals if overheating etc occurs.

$>$ Allow the user to access the data from the internet.

The PC interface, using LabView is also equipped with a tool that allows the user to access the front panel directly from the internet. A portion of the LabView program is shown in fig. 3

\section{Electronic Load}

To obtain a linear load and avoid the switching noise, created by switched resistor loads, the designed electronic load uses power MOSFETs working in the saturation (active) region.

The MOSFET drain current $I_{D}$ is proportional to the voltage $V_{G S}$ applied to the gate and is expressed by equation (1):

$$
I_{D}=k\left(V_{G S}-V_{T H}\right)^{2}
$$

Where $k$, the Gain Factor, depends of the MOSFET geometry, oxide capacitance and carrier mobility. $V_{T H}$ is the threshold voltage [10].

The values of $k$ and $V_{T H}$ are not constant and depend on the MOSFET temperature. Thus, for a given gate voltage the current will drift over time. The influence of $V_{T H}$ drift due to temperature can be neglected for high currents. The designed system avoids output variations by sensing the current and voltage and correcting the gate voltage to maintain the dissipated power at the desired value. 


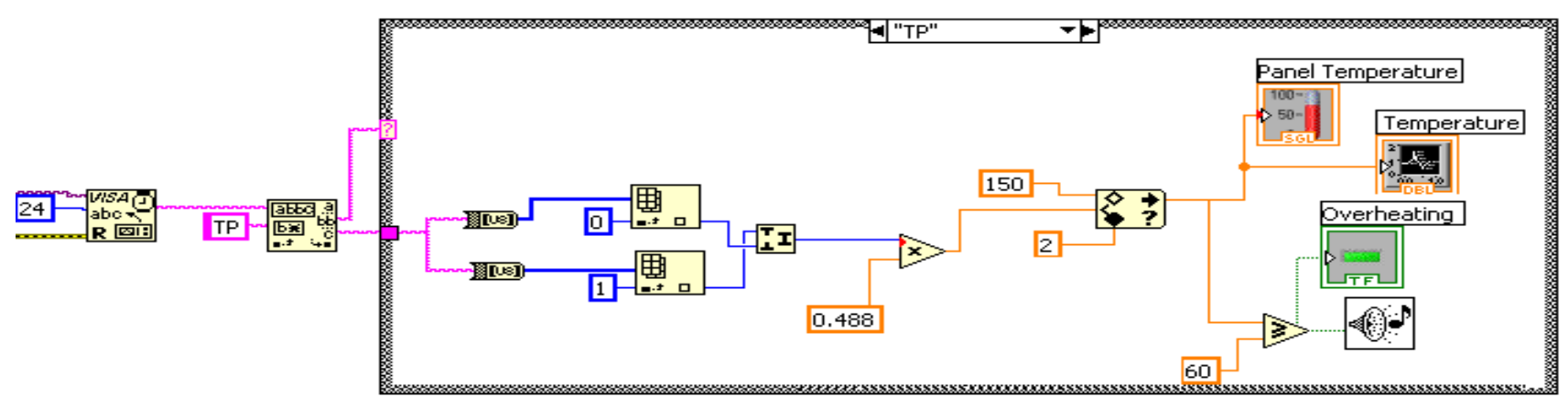

Fig.3: LabView Temperature Conditioning

The control signal is first filtered using a low pass filter to obtain a DC voltage representing the average of the PWM signal and then the signal enters an integrator which controls the power dissipated by the MOSFET. The voltage across the shunt resistor and the voltage between the drain and source of the MOSFET $\left(V_{d s}\right)$ are multiplied and fed in the negative feedback loop, in order to be compared to the control voltage. With this configuration, the non-linearity region of the MOSFET can be accommodated. The block diagram of the load is shown in Fig. 4. An N-channel MOSFET capable of dissipating 50 $\mathrm{W}$ is used which is sufficient to demonstrate the load working principle. If a load with higher dissipation capability is required (to simulate the averaged domestic load profile) the MOSFET can be changed for one with higher dissipation capabilities and paralleled.

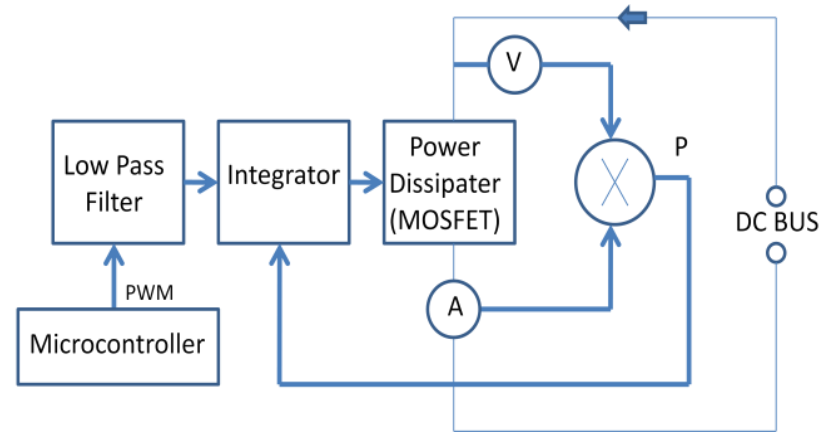

Fig. 4: Electronic Load Block Diagram

The PWM module has a resolution of 1024 bits which provides the load with a step of approximately 50 $\mathrm{mW}$. The PWM frequency is set to $15.5 \mathrm{kHz}$ in order to allow the simulation of load spikes. The program allows the user to select one of the following three modes:

\section{Constant Power:}

Used to simulate a constant load, once selected the user can input the desired power to be dissipated. The power range depends of the load design and on the MOSFET used.

\section{Step:}

Generates user variable current amplitude and time interval steps to test system stability and response.

\section{Household Profile:}

This mode drives the load to emulate the averaged household load profile as shown in Fig. 5.

More load profiles can be added by modification of the microcontroller program.

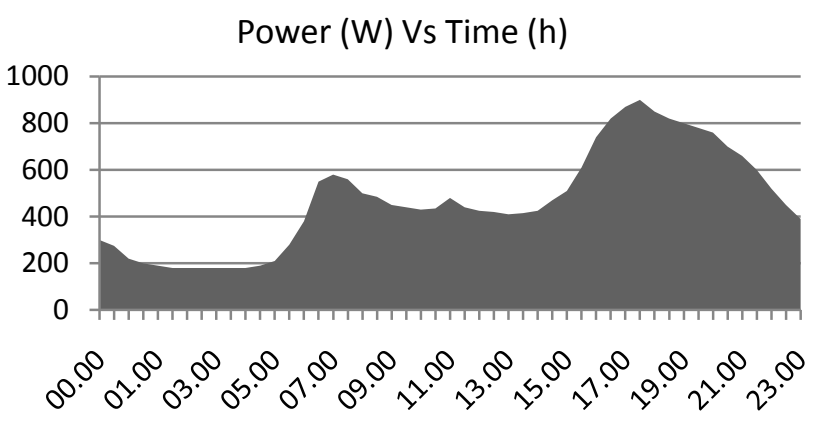

Fig. 5: Typical Winter Domestic Load Profile Produced by the Electricity Association [9]

\section{Results}

The monitoring system was tested using two 20W PV panels connected in parallel. Tests demonstrate that the LCD of the acquisition module displays all useful relevant parameter information and therefore allows easy on-site reading. The transmission link has been successfully tested and the data sent from the acquisition module to the PC, located inside a building, over a range of $50 \mathrm{~m}$. The screenshot, in Fig. 6 shows the temperature and voltage monitoring interface. It also shows the parameter values when the panels were initially exposed to an artificial light source and the temperature started to rise, then the panels placed in shade which allowed the cell temperature to decrease. All the other parameters were also successfully displayed in real time.

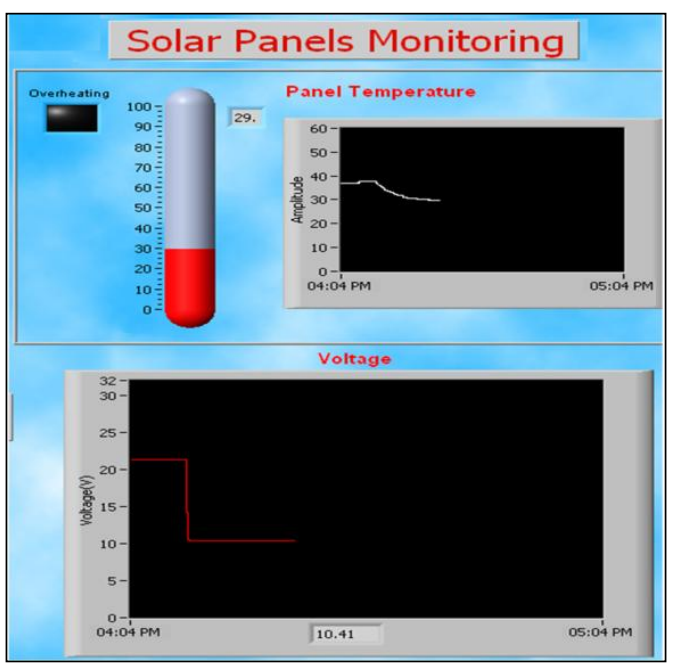

Fig. 6: Voltage and Temperature Monitoring using LabView 


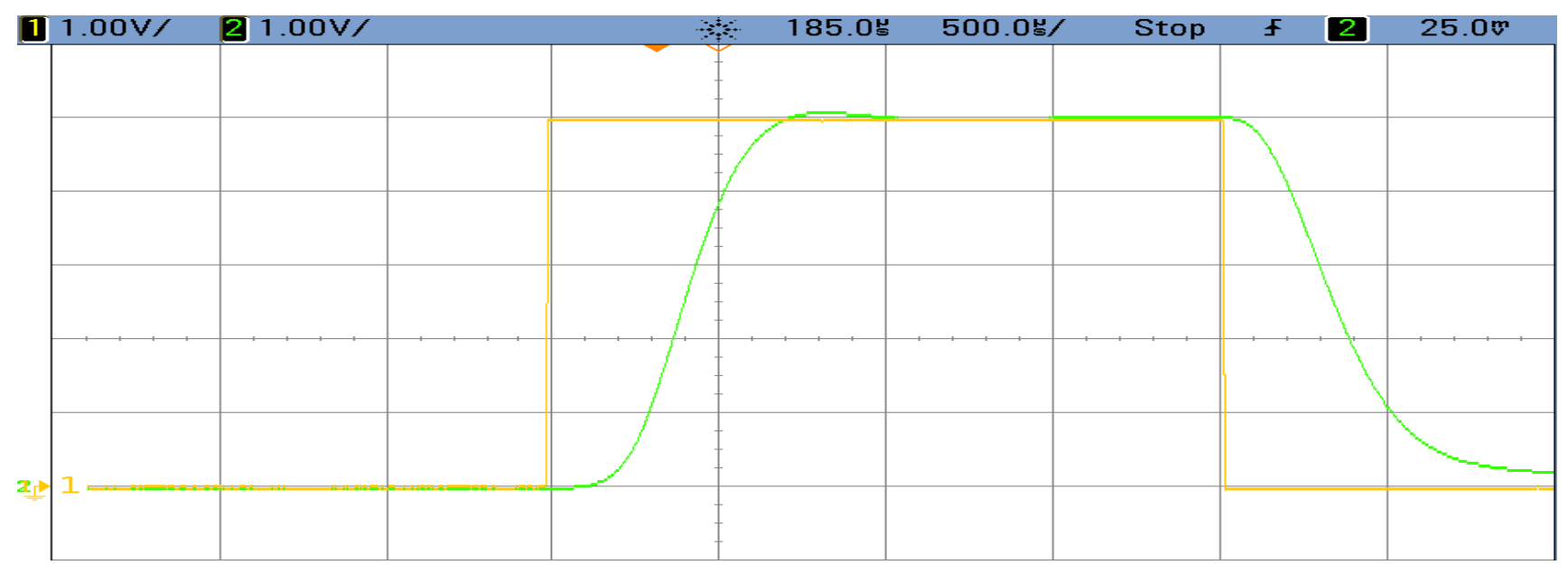

Fig.7: Load Response Time for 0-50W step: Channel 1 Represents the PWM Signal, Channel 2 is the Dissipated Power. $1 \mathrm{~V}=10 \mathrm{~W}$.

The electronic load was connected to a PSU in order to be tested in the laboratory. The control signal of the acquisition module was set to give a $0-100 \%$ duty cycle to yield a $0-50 \mathrm{~W}$ step for the power dissipated by the load. Fig. 8 shows the measurements taken during this test. Channel 1represents the $\mu$ controller output and channel 2 the power dissipated by the electronic load. The load response time is approximately $1 \mathrm{~ms}$ due to the low pass filter cut-off frequency $f_{c}$ being set to approximately $1 \mathrm{kHz}$ to reduce the ripple. After $1 \mathrm{~ms}$ the power is stabilised and is equal to the value set by the control signal. If a faster response is required, $f_{c}$ can be increase along with the PWM frequency or a higher order filter can be used so increase the response time while keeping the ripple to a relatively low value. If the current ripple is not critical and a faster response is required, $f_{c}$ can be increased with no other modifications to the circuit or to the program.

The power ripple of the load is represented in Fig. 8, with channels 1 and 2 representing the PWM signal at the output of the $\mu$ controller and the power dissipated by the load respectively. The channel 3 displays the dissipated power but AC coupled to allow an easy display of the ripple. The main ripple frequency is approximately 15.5 $\mathrm{kHz}$ which is the frequency of the PWM signal. The maximum measured ripple for a $25 \%$ duty cycle is $80 \mathrm{~mW}$ which is $\pm 6 \%$ error. This value is acceptable for RES testing.

\section{Conclusion}

The need for a monitoring and test system for PV based RES installations is addressed and the monitor system being designed and built. The monitoring system is composed of an on-site interface which permits the display of key parameters during the maintenance or modification of the RES. The remote monitoring is also considered and implemented using a Zigbee wireless link for the data transmission and a PC with LabView as a user interface. Finally, an electronic load has been designed to simulate constant, stepped, and typical averaged household load profile conditions in order to test RES.

The results demonstrate that the designed system is able to monitor the current, voltage and temperature of PV panels as well as calculate the output power and energy production. These functionalities make the system a valuable tool to maintain the system efficiency, thus increasing the economical viability. The electronic load was successfully tested and the performances assessed. Although the load is limited to a power dissipation of $50 \mathrm{~W}$, the working principle remains the same if higher power is required.

In the future, the system will be improved with MOSFETs capable of dissipating $1.5 \mathrm{~kW}$ and sensors will be added for fuel cell, hydrogen and weather monitoring.

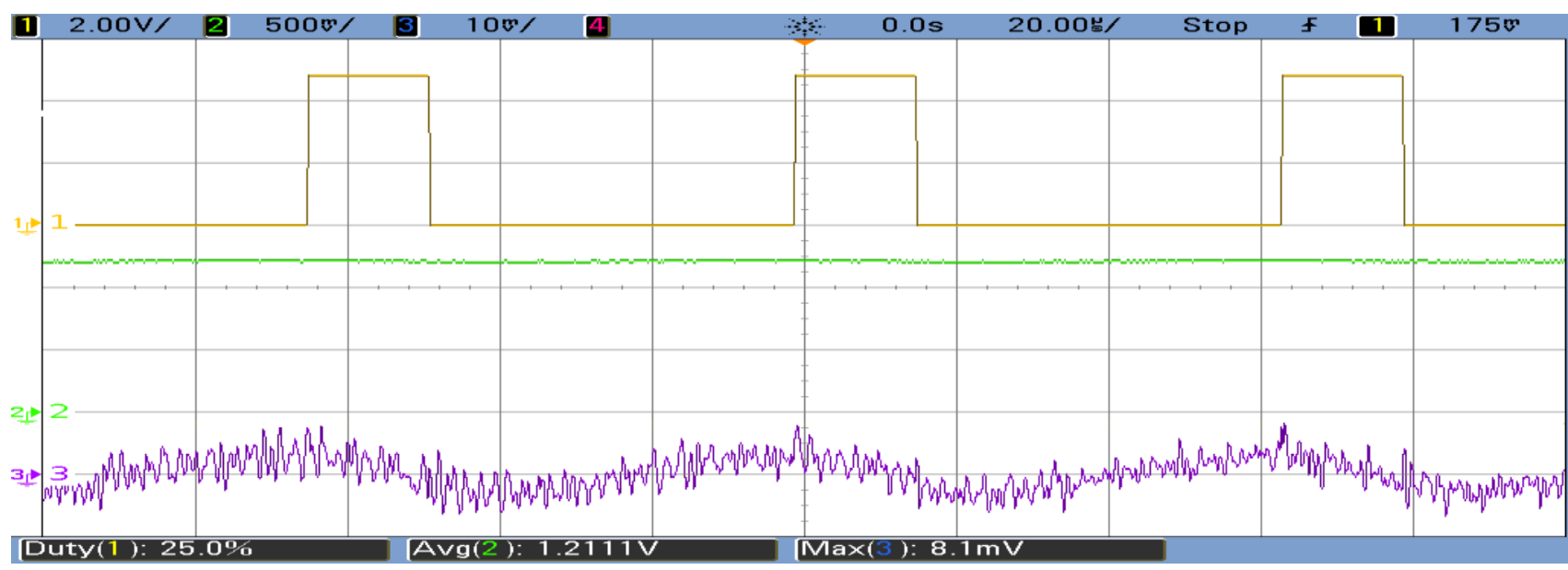

Fig.8: Ripple for a 25\% Duty Cycle (12.5W) Represented on Channel 3 
The system will then be used to develop new control strategies for hybrid RES. The electronic load can be used in any applications that require a flexible and cheap programmable load. (e.g. PSU testing).

\section{Acknowledgement}

The Authors would like to thanks Mr. Chris Daykin for his help and advices throughout the design and test process.

\section{References}

[1] C. Miller and P.N. Edwards, 'Changing the Atmosphere Expert Knowledge and Environmental Governance', Cloth / June 2001.

[2] C. Iskander Yousif, 'Recent Developments of Applying Solar Photovoltaic Technologies in Malta', Proceedings of the 'Enemalta' 25th Anniversary Conference on Energy Efficiency, Mediterranean Conference Centre, 18th-19th October, Valetta, Malta, 2002.

[3] A. Aziz, 'Propriétés Electriques des Composants Electroniques Minéraux et Organiques, Conception et Modélisation d'une Chaîne Photovoltaïque pour une Meilleure Exploitation de l'Energie Solaire', PhD, University Mohamed Premier, N86/06, 2006, Oujda, Morocco, University Paul Sabatier, $N^{\circ} 06234$, Toulouse, France, 2006.

[4] Erik A. Alsema, Mariska J. de Wild-Scholten, Reduction of Environmental Impacts in Crystalline Silicon Photovoltaic Technology, Materials Research Society, Boston, USA (2005)

[5] Øystein Ulleberg, The importance of control strategies in PV-hydrogen systems, Solar Energy 76 (2004) 323329

[6] Calderón M, et al., Automatic management of energy flows of a stand-alone renewable energy supply with hydrogen support, International Journal of Hydrogen Energy (2010), doi:10.1016/j.ijhydene.2009.12.028

[7] Eftichios Koutroulis , Kostas Kalaitzakis, Development of an integrated data-acquisition system for renewable energy sources systems monitoring , Measurement 34 (2003) 75-83.

[8] G.Blaesser, PV system measurements and monitoring: The European experience. Solar energy materials and solar cells 47 (1997) 167-176

[9] S. Abu-Sharkh, Rachel Li, T. Markvart, N. Ross, P. Wilson, R. Yao, K. Steemers, J. Kohler and R. Arnold, Microgrids: distributed on-site generation, Tyndall Centre for Climate Change Research, Technical Report 22 (2005).

[10]P.Horowitz,W.Hill, The art of electronics, Cambridge University press (1989) p.121 